\title{
INTERMEDIATE-TERM PERIODICITIES IN SOFT X-RAY FLARE INDEX DURING SOLAR CYCLES
} 21, 22 AND 23

\author{
BHUWAN JOSHI and ANITA JOSHI \\ Aryabhatta Research Institute of Observational Sciences, Manora Peak, Naini Tal \\ 263 129, Uttaranchal, India (E-mail: bhuwan@aries.ernet.in)
}

\begin{abstract}
We have analyzed the intermediate term periodicities in soft X-ray flare index $\left(F I_{S X R}\right)$ during solar cycles 21, 22 and 23. Power spectral analysis of daily $F I_{S X R}$ reveals a significant period of 161 days in cycle 21 which is absent during cycle 22 and 23. We have found that in cycle 22 periodicities of 74 and 83 days are in operation. A 123 day periodicity has been found to be statistically significant during the part of the current solar cycle 23. The existence of these periodicities has been discussed in the light of earlier results.
\end{abstract}

\section{Introduction}

In the solar cycle 21, an intermediate term (or midrange) periodicity of 154 days was discovered by Rieger et al. (1984) in gamma ray and soft X-ray flare data. Since then a number of studies have been made to look for the evidence of near 155 days periodicity in solar activity. With solar flares and flare related data it has been detected by Bogart and Bai (1985), Ichimoto et al. (1985), Özgüc and Ataç (1989), Dröge et al. (1990), Bai and Cliver (1990), and Kile and Cliver (1991). The presence of this periodicity was also analyzed in several other solar activity features, such as, sunspot area (Lean, 1990; Carbonell and Ballester, 1990, 1992), sunspot blocking function (Lean and Brueckner, 1989), $10.7 \mathrm{~cm}$ radio flux (Lean and Brueckner, 1989), and Photospheric magnetic flux (Ballester, Oliver, and Carbonell, 2002). On the other hand no evidence for the presence of a near 155 days periodicity has been found in any solar activity indicator during solar cycle 22 and part of cycle 23 (Kile and Cliver, 1991; Özgüc and Ataç, 1994; Oliver and Ballester, 1995; Ballester, Oliver, and Carbonell, 2002; Bai, 2003) instead some other prominent periodicities were detected in these studies.

In the present paper, we have made an attempt to investigate the existence of intermediate term periodicities in the soft X-ray flare index $\left(F I_{S X R}\right)$, which is based on the continuous record of soft X-ray (SXR) flares observed by GOES during solar cycles 21, 22, and 23. The $F I_{S X R}$ is calculated by weighing peak intensity of SXR flares of different classes (B to X) in units of $10^{-6} \mathrm{Wm}^{-2}$ (see Joshi and Joshi,

(c) 2014 Kluwer Academic Publishers. Printed in the Netherlands.

bjoshi.tex; 26/01/2014; 2:51; p.1 


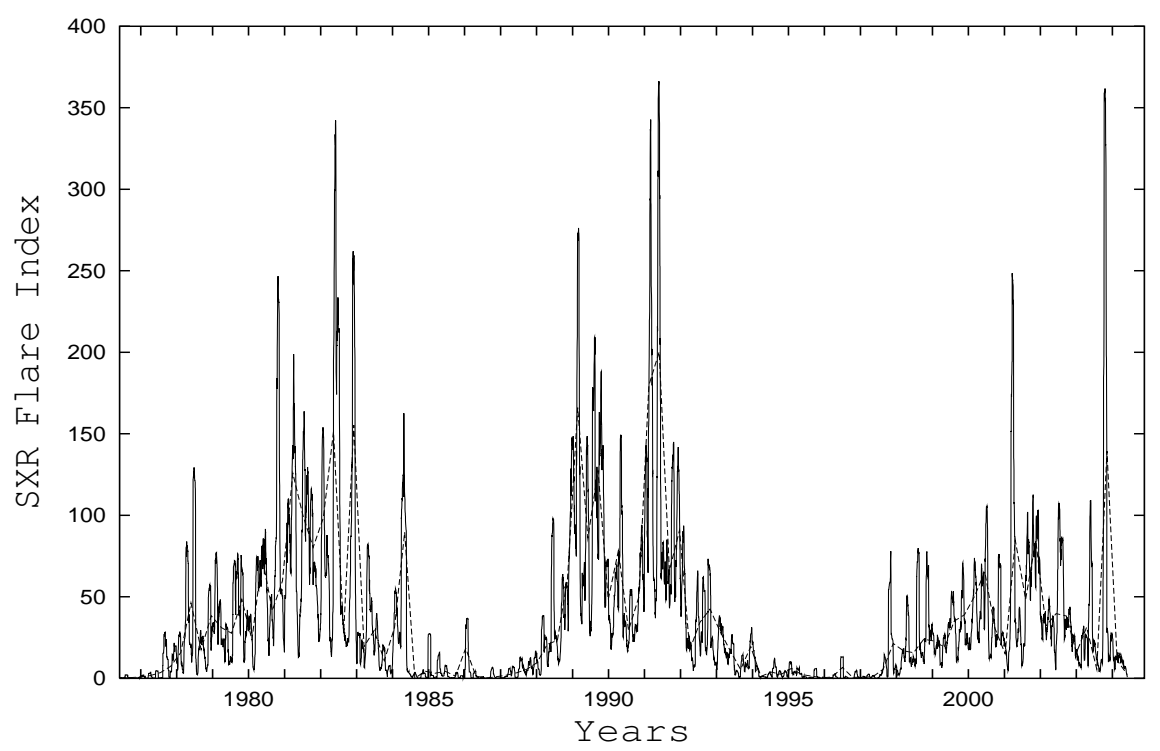

Figure 1. 27-day moving average of daily $F I_{S X R}$ for the period 1976-2004. The dashed line shows the smooth spline curve.

2004 and references therein). In this manner the daily values of $F I_{S X R}$ represent the daily SXR flare activity and are suitable for a short term solar activity analysis. To compute the $F I_{S X R}$, the SXR flare data for the time span of 01 June 1976 to 31 May 2004 has been downloaded from NGDC's anonymous ftp server: ftp://ftp.ng dc.no aa.gov/STP/ SOLAR_DATA/SOLAR_FLARES/XRAY_FLARES. In Figure 1 the time variation of 27-day moving average of daily $F I_{S X R}$ for the period 1976-2004 is presented.

To perform the study, we have split the data in three parts corresponding to the period of solar cycle 21 (June 1976 to August 1986) with 3717 days data, cycle 22 (September 1986 to April 1996) with 3524 days data and cycle 23 (May 1996 to May 2004) with 2953 days data. Cycle 23 is still not complete and the data for this cycle covers the ascending phase, the maximum and part of the descending phase of the solar cycle. Also, for cycle 23 we have performed the power spectra for three different time intervals corresponding to the periods of time studied by Bai (2003).

\section{Periodogram Analysis}

We have Used the Lomb-Scargle periodogram method (Lomb, 1976; Scargle, 1982) modified by Horne and Baliunas (1986) to perform the 

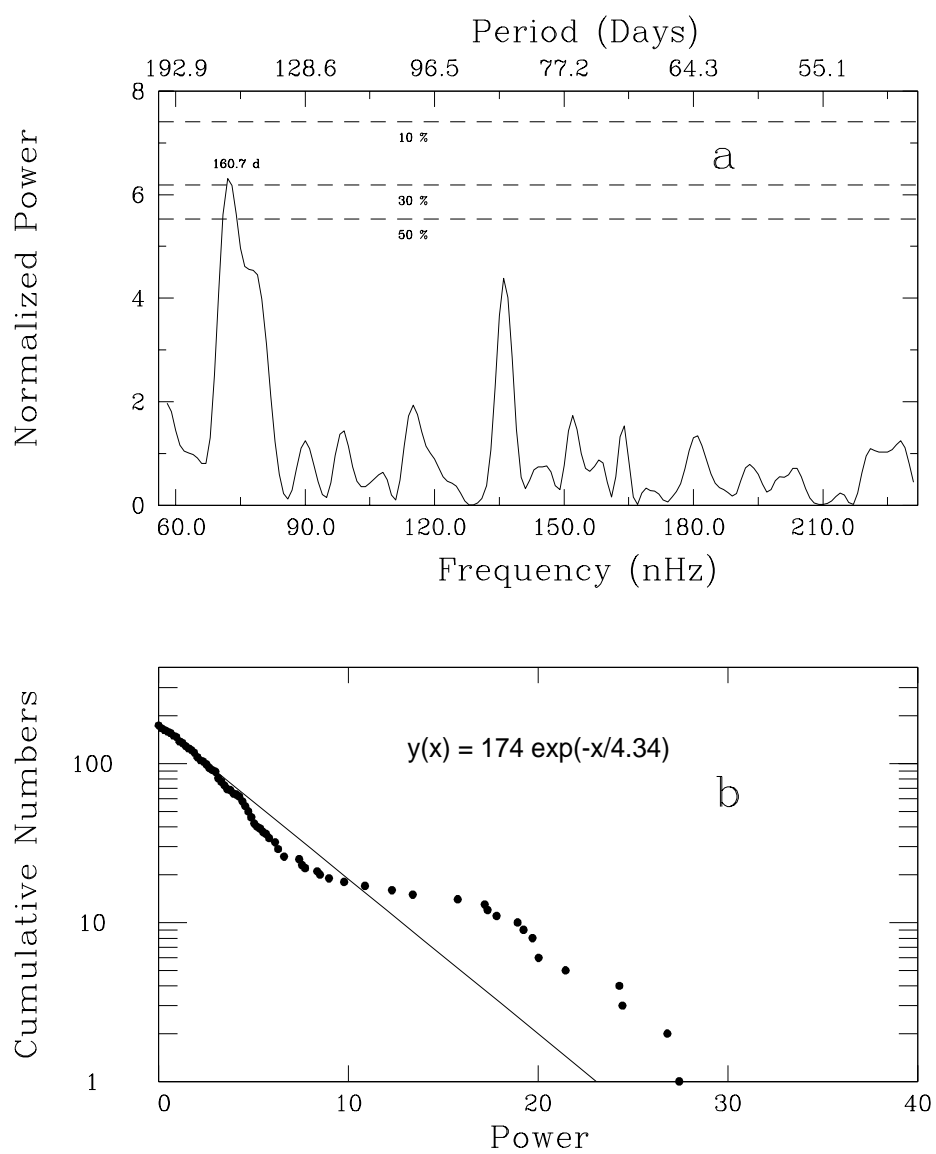

Figure 2. (a) Normalized power spectrum of the daily $F I_{S X R}$ time series of solar cycle 21 for the frequency interval of $231.5-57.8 \mathrm{nHz}$ (50-200 days). Horizontal dashed lines indicate FAP levels of $10 \%, 30 \%$, and $50 \%$ (from top to bottom) (b) Scargle power distribution corresponding to (a). The vertical axis is the number of frequencies for which power exceeds $\mathrm{x}$. The straight line is the fit to the points for lower values of power.

power spectra of $F I_{S X R}$ time series. The power spectra for the three cycles 21,22 and, 23 has been computed for the frequency range of $231.5-57.8 \mathrm{nHz}$ which corresponds to the period interval of $50-200$ days. Figure 2(a) shows the normalized power spectra of daily $F I_{S X R}$ values for solar cycle 21 . The $F I_{S X R}$ is not independent. Therefore the probability $\mathrm{P}$ of the power density at a given frequency being greater than $\mathrm{K}$ by chance is given by

$$
P(z>K)=\exp (-K / k)
$$



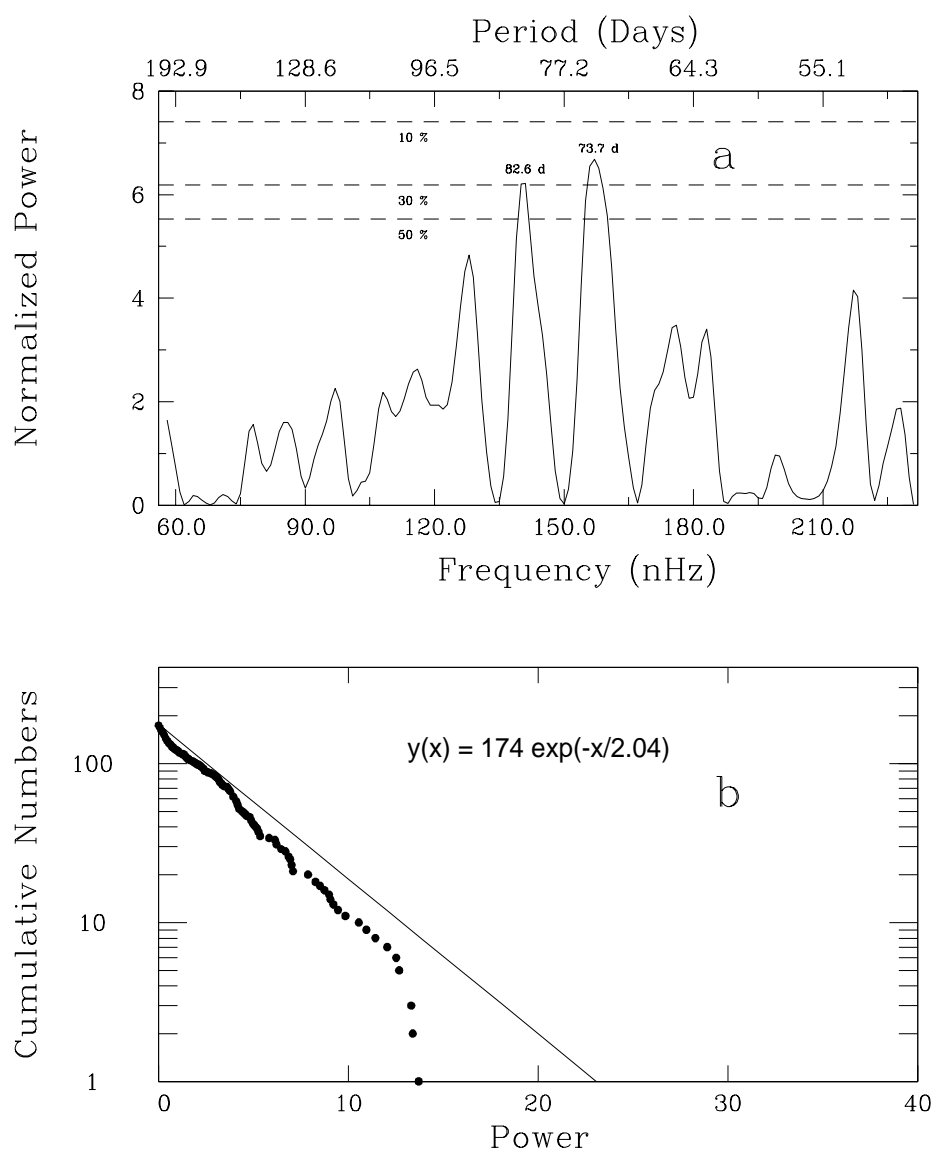

Figure 3. Same as Figure 1, but for solar cycle 22.

where the normalization factor $k$, which is due to event correlation, can be determined empirically (Bai and Cliver, 1990).

Figure 2(b) shows the distribution of the power values corresponding to the normalized power spectra shown in Figure 2(a). The vertical axis shows the cumulative number of frequencies for which the power exceeds a certain value. For example, in the power spectrum for solar cycle 21 shown in Figure 2(a) we have 174 frequencies. For all these frequencies the power exceeds zero; thus, we have a point at $(\mathrm{X}=0$, $\mathrm{Y}=174)$. At only one frequency $(72.0 \mathrm{nHz}$, which is equivalent to 160.75 days) the power was 27.42, its maximum value. For lower values of power, the distribution can be well fitted by the equation $\mathrm{y}=174$ $\exp (-\mathrm{x} / 4.34)$, as expected from equation (1). Thus, we normalize the power spectrum by dividing the powers by 4.34 to obtain Figure 2(a). For other cases we use the same procedure for normalization. 

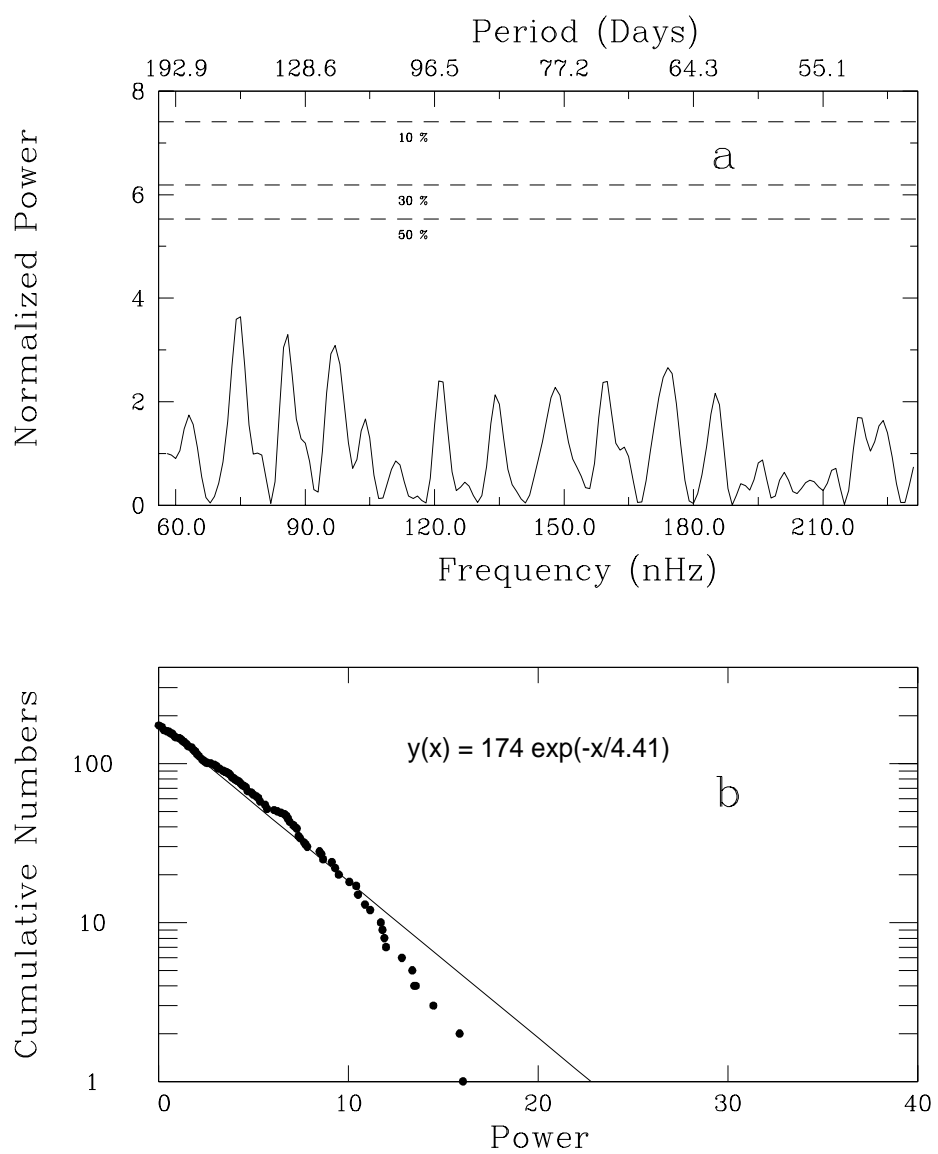

Figure 4. Same as Figure 1, but for solar cycle 23.

Once the power spectrum has been normalized properly, we can use the 'False alarm probability' (FAP) formula to estimate the statistical significance of a peak in the power spectrum. It is given by the expression

$$
F=1-\left[1-\exp \left(-Z_{m}\right)\right]^{N},
$$

where $Z_{m}$ is the height of the peak in the normalized power spectrum and $N$ is the number of independent frequencies. The interpretation of $F$ is as follows: if we have a discrete power spectrum giving the power at each of $N$ independent frequencies for a set of random data, $F$ indicates the probability that the power at one or more of these frequencies will exceed $Z_{m}$ by chance. Fourier components calculated at frequencies at intervals of independent fourier spacing, $\Delta f_{\text {ifs }}=\tau^{-1}$, where $\tau$ is the time span of the data, are totally independent (Scargle, 1982). For example, in the power spectrum shown in Figure 2(a), we 

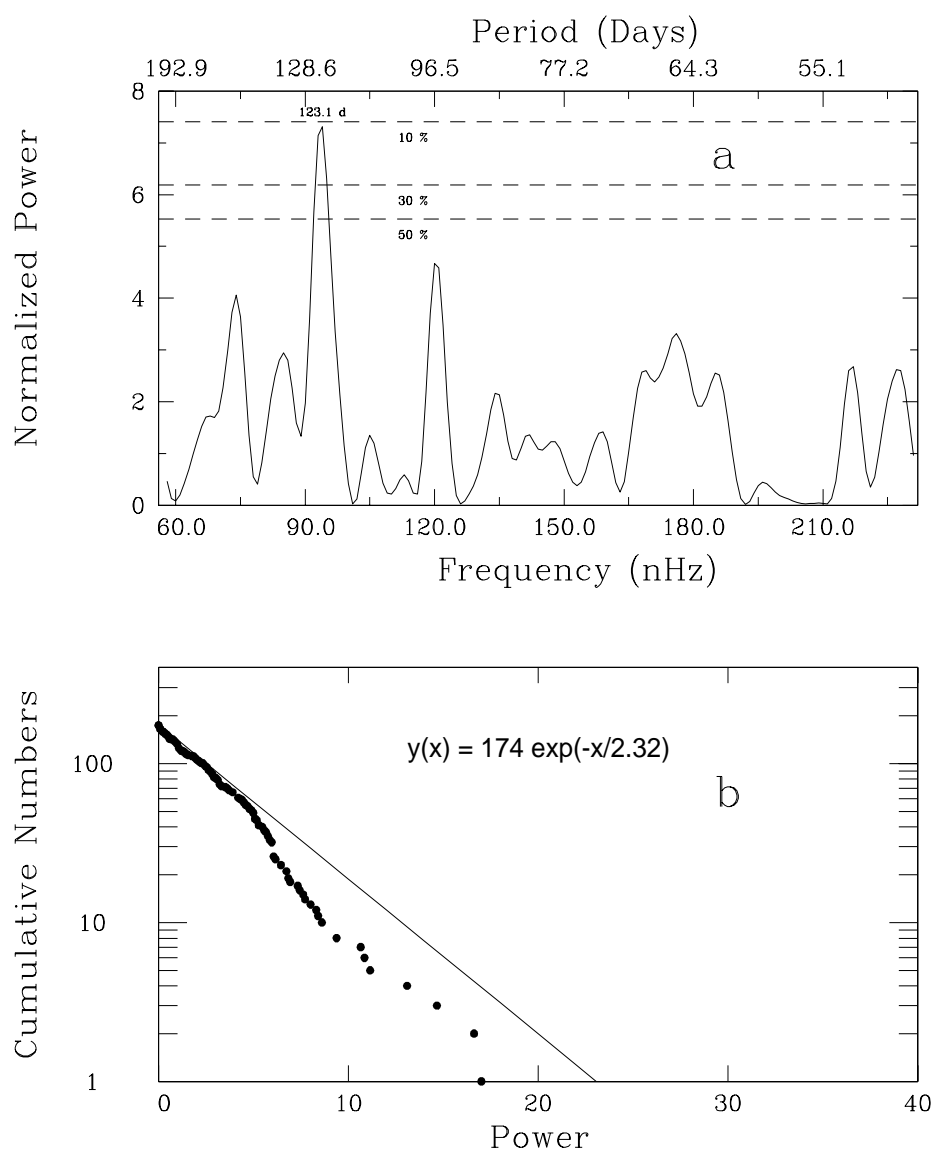

Figure 5. Same as Figure 1, but for the interval from May 1996 to October 2002 of the solar cycle 23 .

have $\tau=3717$ days and $\Delta f_{i f s}=3.1 \mathrm{nHz}$. Thus there are 56 independent frequencies in $231.5-57.8 \mathrm{nHz}$ interval. We have oversampled to obtain this power spectrum in which the height of the peak at 160.75 days is 6.3 . The oversampling tends to estimate more accurately the peak value. Therefore, if we substitute $Z_{m}=6.3$ and $\mathrm{N}=174$ (since we searched 174 frequencies with $1.0 \mathrm{nHz}$ intervals) in equation (2) we get $F=0.27$, i.e., the probability to obtain such a high peak at 72.0 $\mathrm{nHz}$ (160.75 days) by chance is about $27 \%$. The same analysis has been applied to the other power spectra also shown in Figure (3), (4), and (5). In the power spectra for solar cycle 22 (Figure 3 ) we have found two important peaks at $157.0 \mathrm{nHz}$ (73.72 days) with $\mathrm{FAP}=19$ $\%$ and $140.4 \mathrm{nHz}$ (82.67 days) with $\mathrm{FAP}=32.9 \%$. No significant peak appears in the power spectra for solar cycle 23 (Figure 4) when the 
data from the beginning of cycle 23 to 31 May 2004 was analyzed. We have also performed the power spectra for three different time intervals (May 1996 to October 2002, September 1999 to June 2001 and October 1999 to April 2001) studied by Bai (2003). We could not find any statistically significant peak in the second and third duration. However in the first time interval (i.e., May 1996 to October 2002) a significant peak appears at $94.0 \mathrm{nHz}$ (123.13 days) with $\mathrm{FAP}=11 \%$ (Figure 5 ).

To be sure that peaks in the different power spectra (Figures 2, 3 , and 5) are not due to aliasing, we have removed sine curves of their periods from the original time series. We see that these peaks get removed in the power spectra of time series obtained after subtraction.

\section{Summary and Discussion}

We have studied periodicities in $F I_{S X R}$ during solar cycles 21,22 and 23 separately. It will be useful to compare and discuss our results in the light of periodicities detected in other solar activity indicators.

We have found that a period of 161 days appeared in $F I_{S X R}$ during solar cycle 21, which is absent during cycle 22 and 23. The near 155 days periodicity, first detected by Rieger et al. (1984) in gamma ray and soft X-ray flares during cycle 21, has been found in several other studies. During the same solar cycle, it was detected in flare producing energetic interplanetary electrons (Dröge et al., 1990), proton flares (Bai and Cliver, 1990), and microwave flares (Bogart and Bai, 1985; Kile and Cliver, 1991). This periodicity was also found in $\mathrm{H} \alpha$ flares (Ichimoto et al., 1985), microwave flares (Bogart and Bai, 1985), and $\mathrm{H} \alpha$ flare index (Özgüc and Ataç, 1989) when the data of solar cycles 20 and 21 was considered together. Lean and Brueckner (1989) detected a periodicity at 155 days in the sunspot blocking function and the $10.7 \mathrm{~cm}$ radio flux during solar cycles 19, 20 and 21. Carbonell and Ballester $(1990,1992)$ have shown that a periodicity around 150-160 days seems to be significant during all solar cycles from 16 to 21 in sunspot areas. Recently Ballester, Oliver, and Carbonell (2002) in the analysis of historical record of photosphere magnetic flux detected a significant periodicity near 160 days during solar cycle 21 .

Several mechanism have been put forward to explain the cause of near 155 days periodicity. Bai and Sturrock (1991) and Sturrock and Bai (1992) proposed that Sun contains a "clock" with a period of 25.8 days (later modified as 25.5 days) and suggested that periodicity of 154 days is a subharmonic of that fundamental period. Bai and Sturrock (1993) analyzed the longitude distribution of major flares of cycles 19-22 and interpreted this hypothetical clock as being an obliquely

bjoshi.tex; 26/01/2014; 2:51; p.7 
rotating structure (or a wave pattern) rotating with a period of 25.5 days about an axis titled by $40^{\circ}$ with respect to the solar rotation axis. On the other hand, another explanation suggests that this periodicity is associated with the periodic emergence of the magnetic flux linked to the regions of strong magnetic field (Carbonell and Ballester, 1990, 1992; Oliver, Ballester, and Baudin, 1998; Ballester, Oliver, and Baudin, 1999; Ballester, Oliver, and Carbonell, 2002).

Other periodicities detected by us in $F I_{S X R}$ during cycles 22 and 23 are also well consistent with the earlier work. We detected 74 days and 83 days periodicities in $F I_{S X R}$ for cycle 22 . Bai (1992) analyzed the major flare occurrence of solar cycle 22 and found a periodicity of 77 days which was interpreted as the third subharmonic of the 25.5 days period. Özgüc and Ataç (1994) also found a period of 73 days in $H \alpha$ flare index during cycle 22. Oliver and Ballester (1995), in the analysis of sunspot areas, found a periodicity around 86 days (close to 83 days period in $\left.F I_{S X R}\right)$ which is statistically significant during part of solar cycle 22 . For cycle 23 we have carried out analysis in four different time intervals, but a significant peak appears only in the duration of May 1996 to October 2002 with a period of 123 days. Lou et al. (2003) found a periodicity of 122 days in X-ray flares of class $\geq$ M5.0 during the maximum phase of solar cycle 23. Bai (2003) detected a periodicity of 129 days in major flares during part of cycle 23 (September 1999 to June 2001). Here it is interesting to note that 74 and 123 days periodicities, found in $F I_{S X R}$, are close to integral multiple of 25.5 days.

\section{Acknowledgements}

We are thankful to Prof. Ram Sagar for valuable suggestions. One of the authors (BJ) wishes to thank Dr. Brijesh Kumar for useful discussions. We thank an anonymous referee for helpful comments and suggestions. BJ also wishes to thank Dr. P. Pant for several useful discussions.

\section{References}

Bai, T.: 1992, Astrophys. J. 388, L69.

Bai, T.: 2003, Astrophys. J. 591, 406.

Bai, T. and Cliver, E. W.: 1990, Astrophys. J. 363, 299.

Bai, T. and Sturrock, P. A.: 1991, Nature 350, 141.

Bai, T. and Sturrock, P. A.: 1993, Astrophys. J. 409, 476.

Ballester, J. L., Oliver, R., and Baudin, F.: 1999, Astrophys. J. 522, L153. 
Ballester, J. L., Oliver, R., and Carbonell, M.: 2002, Astrophys. J. 566, 505.

Bogart, R. S. and Bai, T.: 1985 Astrophys. J. 299, L51.

Carbonell, M. and Ballester, J. L.: 1990, Astron. Astrophys. 238, 377.

Carbonell, M. and Ballester, J. L.: 1992, Astron. Astrophys. 255, 350.

Dröge, W., Gibbs, K., Grunsfeld, J. M., Meyer, P., Newport, B. J., Evenson, P., and Moses, D.: 1990, Astrophys. J. Supll. Ser. 73, 279.

Horne, J. H. and Baliunas, S. L.: 1986, Astrophys. J. 302, 757.

Ichimoto, K., Kubota, J., Suzuki, M., Tohmura, I., and Kurokawa, H.: 1985, Nature 316, 422.

Joshi, B. and Joshi, A.: 2004, Solar Phys. 219, 343.

Kile, J. N. and Cliver, E. W.: 1991, Astrophys. J. 370, 442.

Lean, J. L.: 1990 Astrophys. J. 363, 718.

Lean, J. L. and Brueckner, G. E.: 1989 Astrophys. J. 337, 568.

Lomb, N.: 1976, Astrophys. Space Sci. 39, 477.

Lou, Y., Wang, Y., Fan, Z., Wang, S., and Wang, J.: 2003, Monthly Notices Royal Astron. Soc. 345, 809.

Oliver, R., Ballester, J.L.: 1995, Solar Phys. 156, 145.

Oliver, R., Ballester, J. L., and Baudin, F.: 1998, Nature 394 , 552.

Özgüç, A. and Ataç, T.: 1989, Solar Phys. 123, 357.

Özgüç, A. and Ataç, T.: 1994, Solar Phys. 150, 339.

Rieger, E., Share, G. H., Forrest, D. J., Kanbach, G., Reppin, C., and Chupp, E. L.: 1984, Nature 312, 623.

Scargle. J. D.: 1982, Astrophys. J. 263, 835.

Sturrock, P. A. and Bai, T.: 1992, Astrophys. J. 397, 337.

Address for Offprints: Bhuwan Joshi

Aryabhatta Research Institute of Observational Sciences (ARIES),

Manora Peak, Naini Tal 263 129,

INDIA.

Phone: +91-05942-235583, 235136

Fax: +91-05942-235136

email: bhuwan@upso.ernet.in 
bjoshi.tex; 26/01/2014; 2:51; p.10 